\title{
HER2-positive breast cancer patients receiving trastuzumab treatment obtain prognosis comparable with that of HER2-negative breast cancer patients
}

\author{
This article was published in the following Dove Press journal: \\ OncoTargets and Therapy \\ 8 April 2013 \\ Number of times this article has been viewed
}

\author{
Tao Qin* \\ Zhongyu Yuan* \\ Roujun Peng \\ Bing Bai \\ Yanxia Shi \\ Xiaoyu Teng \\ Donggeng Liu \\ Shusen Wang \\ Department of Medical Oncology, \\ State Key Laboratory of Oncology \\ in South China, Sun Yat-sen University \\ Cancer Center, Guangzhou, People's \\ Republic of China \\ *These authors contributed equally \\ to this work
}

Correspondence: Shusen Wang Department of Medical Oncology, State Key Laboratory of Oncology in South China, Sun Yat-sen University Cancer Center, 65I East Dongfeng Road, Guangzhou 510060, Guangdong, People's Republic of China Tel +862087343794 Email wangshs@sysucc.org.cn
Purpose: The efficacy of trastuzumab in Chinese breast cancer (BC) patients has rarely been reported. This study was designed to compare the clinical outcomes of HER2-positive BC patients receiving or not receiving trastuzumab treatment and HER2-negative BC patients.

Patients and methods: This study involved three groups of patients. The first group was 115 human epidermal growth factor receptor 2 (HER2)-positive BC patients treated with trastuzumab who were enrolled at Sun Yat-sen University Cancer Center between January 2002 and July 2010; the second group was a matched control group of 115 HER2-positive patients who did not receive trastuzumab treatment; the third group was a matched group of 115 HER2-negative patients who received conventional therapy in the adjuvant setting. The primary endpoint was 3-year and 5-year disease-free survival (3-DFS and 5-DFS, respectively). The Kaplan-Meier method, log-rank test, and multivariate Cox proportional hazard regression model were used for survival analysis. The differences in survival rates among the three groups were also analyzed according to two different periods: 2002-2006 and 2007-2010.

Results: The median duration of follow-up was 36 months (range, 12-111 months). The 3-DFS rates in the HER2-negative group, the HER2-positive group who received trastuzumab treatment, and the HER2-positive group who did not receive trastuzumab treatment were $82.6 \%$, $89.6 \%$, and $67.0 \%$, respectively. The 3-DFS rate for the total study population was statistically significant $(P<0.001)$. Further analysis indicated a statistically significant difference in 3-DFS between either of the first two groups and the third group $(P<0.01)$, but the difference between the first two groups was not statistically significant $(P=0.157)$. Among the three groups, the 3-DFS rates during 2002-2006 did not have a significant difference compared with that during 2007-2010.

Conclusion: This study has further confirmed the efficacy of trastuzumab for HER2-positive operable BC in Chinese patients. It has also demonstrated that the 3-DFS and 5-DFS rates between HER2-positive patients receiving trastuzumab treatment and HER2-negative patients are comparable.

Keywords: breast cancer, HER2, trastuzumab, adjuvant treatment, survival analysis

\section{Introduction}

Breast cancer (BC) has become the most common malignancy and the leading cause of cancer-specific death in women, according to GLOBOCAN 2008 estimates. ${ }^{1}$ With an increasing understanding of the biological behavior of $\mathrm{BC}$, especially in relation to molecular-based personalized medicine for $\mathrm{BC}$, the concept of $\mathrm{BC}$ treatment has been revolutionized, resulting in improved survival rates and quality of life..$^{2-4}$ 
A typical example of this increase in understanding is the pivotal role of human epidermal growth factor receptor 2 (HER2), also known as c-erbB-2 or HER2/neu, a key member of the epidermal growth factor receptor family overexpressed in approximately $20 \%-25 \%$ of invasive BC. ${ }^{5-8}$ HER2-overexpression or HER2/neu gene amplification (defined as HER2 positive) has been shown in BC patients to be closely associated with the more aggressive phenotype and to be highly metastasized to regional lymph nodes and distant organs (this has been used as an indicator of poor prognosis). ${ }^{5-8}$

With an increasing understanding of the role of HER 2 in tumor proliferation, angiogenesis, and metastasis, novel special treatment strategies for this HER2-positive subtype of $\mathrm{BC}$ have been validated and are increasingly used in clinical practice. One of the most important treatment strategies is to block the signal pathway of HER2/neu - this is defined as targeted therapy. Trastuzumab (Herceptin ${ }^{\circledR}$ ), a humanized monoclonal antibody, is a typical agent for anti-HER2 treatment and has been developed for $\mathrm{BC}$ treatment over the past 10 years..$^{5-8}$

Several large clinical trials have shown that the adjuvant treatment of trastuzumab in HER2-positive BC patients resulted in a $30 \%-50 \%$ relative risk reduction of recurrence in adjuvant settings, ${ }^{9-11}$ as well as an increased pathologic complete remission rate in neoadjuvant settings. ${ }^{12-16}$ Although the HER2-targeted therapy has progressed considerably since 1998, the efficacy of trastuzumab in Chinese BC patients has rarely been reported, especially as part of a comparison with HER2-negative BC patients.

The purpose of this study was to compare the clinical outcomes of HER2-positive BC patients receiving or not receiving trastuzumab treatment and HER2-negative $\mathrm{BC}$ patients, in a retrospective analysis of operable $\mathrm{BC}$ patients in the People's Republic of China.

\section{Patients and methods Patients and study design}

All eligible patients were diagnosed with invasive BC and treated at Sun Yat-sen University Cancer Center, People's Republic of China, between January 2002 and July 2010. Patients were enrolled based on the following criteria: (1) the invasive $\mathrm{BC}$ was confirmed and classified based on the pathologic examination; (2) evaluation of hormone receptors and HER2 status; (3) complete medical records (including types of surgery and adjuvant treatments) and follow-up status; (4) no previous diagnosis and treatment of BC; (5) stage I-III prior to neoadjuvant chemotherapy or following surgery, based on the American Joint Committee on Cancer staging system (sixth edition); and (6) an Eastern Cooperative Oncology Group performance status score of $0-2$. Patient characteristics are summarized in Table 1.

To begin, 115 HER2-positive BC patients receiving trastuzumab treatment were enrolled in the study. Two groups matched in year of diagnosis, age, family history of $\mathrm{BC}$, tumor size, lymph node status, cancer stage, expression of hormone receptor, amplification of HER2, and treatment (surgery, chemotherapy, endocrine treatment, or radiotherapy) were also selected: 115 case-control HER2-positive patients who did not receive trastuzumab treatment and 115 HER2-negative patients who received conventional therapy in the adjuvant setting. All patients in the study were treated with standard adjuvant chemotherapy and endocrine therapy strategies according to National Comprehensive Cancer Network guidelines. The duration of the adjuvant endocrine therapy was planned for 5 years. The cycles of chemotherapy depended on standard regimen that followed National Comprehensive Cancer Network guidelines.

This study was reviewed and approved by the Institutional Review Board of Sun Yat-sen University Cancer Center.

\section{HER2 evaluation and treatment}

HER2 status was initially evaluated using immunohistochemistry (IHC). IHC results showing a 2+ HER2 level were further tested by fluorescence in situ hybridization. The patients showing a 3+ HER2 level by IHC and/or HER2/neu gene amplification by fluorescence in situ hybridization were described as being HER2-positive. The HER2-positive patients in the group that received trastuzumab received an initial dose of $8 \mathrm{mg} / \mathrm{kg}$, followed by $6 \mathrm{mg} / \mathrm{kg}$ every 3 weeks, or an initial dose of $4 \mathrm{mg} / \mathrm{kg}$, followed by $2 \mathrm{mg} / \mathrm{kg}$ weekly, following or concurrent with chemotherapy. The duration of trastuzumab treatment was set at 1 year. Other major treatment information, including types of surgery and adjuvant treatments (chemotherapy, radiotherapy, and endocrine therapy), was obtained from the patients' medical records. All of the patients with hormone receptor-positive $\mathrm{BC}$, which can be further defined as estrogen receptor and/or progesterone receptor positive, received endocrine therapy. The patient treatment strategies are summarized in Table 1.

\section{Patient follow-up}

All patients in this study were on a regular follow-up schedule. The primary endpoint was disease-free survival, 
Table I Baseline clinical and pathologic characteristics of patients

\begin{tabular}{|c|c|c|c|c|}
\hline \multirow[t]{2}{*}{ Characteristic } & \multirow{2}{*}{$\begin{array}{l}\text { HER2-negative } \\
\text { patients }^{\mathrm{a}} \text { [n (\%)] }\end{array}$} & \multicolumn{2}{|l|}{ HER2-positive patients } & \multirow[t]{2}{*}{$P$-value } \\
\hline & & Trastuzumab $^{\mathrm{a}}$ [n (\%)] & Non-trastuzumab ${ }^{\mathrm{a}}[\mathrm{n}(\%)]$ & \\
\hline \multicolumn{5}{|l|}{ Age (years) } \\
\hline$\leq 50$ & $74(64.3)$ & $74(64.3)$ & $73(64.1)$ & 0.987 \\
\hline$>50$ & $4 \mathrm{I}(35.7)$ & $4 \mid(35.7)$ & $42(35.9)$ & \\
\hline \multicolumn{5}{|l|}{ Tumor size } \\
\hline $\mathrm{TI}^{\mathrm{b}}$ & $31(27.0)$ & $46(40.0)$ & $34(29.6)$ & 0.033 \\
\hline $\mathrm{T} 2^{\mathrm{c}}$ & $76(66.1)$ & $59(5 \mathrm{I} .3)$ & $63(54.8)$ & \\
\hline$T 3^{d}$ & $8(7.0)$ & $10(8.7)$ & $18(15.7)$ & \\
\hline \multicolumn{5}{|l|}{ Tumor grade } \\
\hline 1 & $14(12.2)$ & $17(14.8)$ & II (9.6) & 0.767 \\
\hline 2 & $73(63.5)$ & $74(64.3)$ & $75(65.2)$ & \\
\hline 3 & $28(24.3)$ & $24(20.9)$ & $29(25.2)$ & \\
\hline \multicolumn{5}{|l|}{ Lymph node status } \\
\hline 0 & $46(40.0)$ & $46(40.0)$ & $37(32.2)$ & 0.793 \\
\hline $1-3$ & $32(27.8)$ & $32(27.8)$ & $4 \mathrm{I}(35.7)$ & \\
\hline $4-9$ & $24(20.9)$ & $24(20.9)$ & $26(22.6)$ & \\
\hline$>9$ & $13(11.3)$ & $13(11.3)$ & II (9.6) & \\
\hline \multicolumn{5}{|l|}{ HR status } \\
\hline Positive & $72(62.6)$ & $70(60.9)$ & $69(60.0)$ & 0.918 \\
\hline Negative & $43(37.4)$ & $45(39.1)$ & $46(40.0)$ & \\
\hline \multicolumn{5}{|l|}{ Surgery type } \\
\hline Mastectomy & $104(90.4)$ & $107(93.0)$ & $106(91.9)$ & 0.762 \\
\hline Lumpectomy & II (9.6) & $8(7.0)$ & $9(8.1)$ & \\
\hline \multicolumn{5}{|l|}{ Radiotherapy } \\
\hline Yes & $34(29.6)$ & $54(47.0)$ & $36(31.3)$ & 0.01 \\
\hline No & $81(70.4)$ & $61(53.0)$ & $79(68.7)$ & \\
\hline \multicolumn{5}{|l|}{ Endocrine therapy } \\
\hline Yes & $72(62.6)$ & $70(60.9)$ & $69(60.0)$ & 0.918 \\
\hline No & $43(37.4)$ & $45(39.1)$ & $46(40.0)$ & \\
\hline \multicolumn{5}{|l|}{ Chemotherapy } \\
\hline Yes & $106(92.2)$ & $113(98.3)$ & $105(91.3)$ & 0.056 \\
\hline No & $9(7.8)$ & $2(1.7)$ & $10(8.7)$ & \\
\hline Chemotherapy regimen & & & & 0.998 \\
\hline CMF & $6(5.2)$ & $7(6.1)$ & $6(5.2)$ & - \\
\hline TC & $8(7.0)$ & $10(8.7)$ & $7(6.1)$ & - \\
\hline CAF & $28(24.3)$ & $31(27.0)$ & $29(25.2)$ & - \\
\hline AC-T/TAC & $64(55.7)$ & $65(56.5)$ & $63(54.8)$ & - \\
\hline
\end{tabular}

Notes: ${ }^{a} \mathrm{n}=115$; ${ }^{b}$ tumor is not larger than $2 \mathrm{~cm}$; ${ }^{\mathrm{c} t u m o r}$ is larger than $2 \mathrm{~cm}$ but not larger than $5 \mathrm{~cm}$; ${ }^{\mathrm{d}}$ tumor is larger than $5 \mathrm{~cm}$.

Abbreviations: HER2, human epidermal growth factor receptor 2; HR, hormone receptor; CMF, cyclophosphamide, methotrexate, and fluorouracil; TC, docetaxel and cyclophosphamide; CAF, cyclophosphamide, doxorubicin, and fluorouracil; AC-T, doxorubicin and cylophosphamide followed by paclitaxel; TAC, docetaxel, doxorubicin, and cyclophosphamide.

defined as the interval of time from the date of BC surgery to the first evidence of recurrence (local, regional, or distant) or death from any cause which came first. Median follow-up time was calculated as the median observation period for the total study population. If recurrence was not evident, patients were censored on the final follow-up date. Because the recurrence of $\mathrm{BC}$ commonly occurs within the 3 years following initial diagnosis, especially for HER2-positive BC patients, the authors selected 3-DFS as the primary endpoint for efficacy evaluation among the three groups in this study. The authors also divided the study period into two separate intervals, 2002-2006 and 2007-2010, to compare the efficacy shown in these different periods. The 5-year disease-free survival (5-DFS) rates among the three groups during the 2002-2006 period were also analyzed.

\section{Statistical analysis}

Pearson's Chi-square test was used to compare clinicopathologic parameters of patients among the groups. The Kaplan-Meier method was used to determine the 3-DFS or 5-DFS probability, and the differences were assessed using the log-rank test. The multivariate Cox proportional hazard regression model was used to evaluate the independent prognostic factors. Hazard ratios with $95 \%$ confidence intervals 
were also obtained. All statistical analyses were performed using SPSS software, version 16.0 (SPSS Inc, Chicago, IL, USA), and a two-tailed $P$-value less than 0.05 was considered statistically significant.

\section{Results}

The range of ages in the HER2-positive group was 26-71 years (median age, 46 years), and in the HER2-negative group the range was $25-70$ years (median age, 46 years). The main clinicopathologic characteristics of the HER2-negative patients were well matched with those of the HER2-positive patients.

The median follow-up period was 36 months (range, 12-111 months). The median durations of follow-up for the periods 2002-2006 and 2007-2010 were 55 months (range, $37-111$ months), and 25 months (range, 12-36 months) respectively. The 3-DFS was selected as the primary endpoint, with 35 local recurrences and 35 distant recurrences. The 3-DFS rates in the HER2-negative group, the HER2-positive group
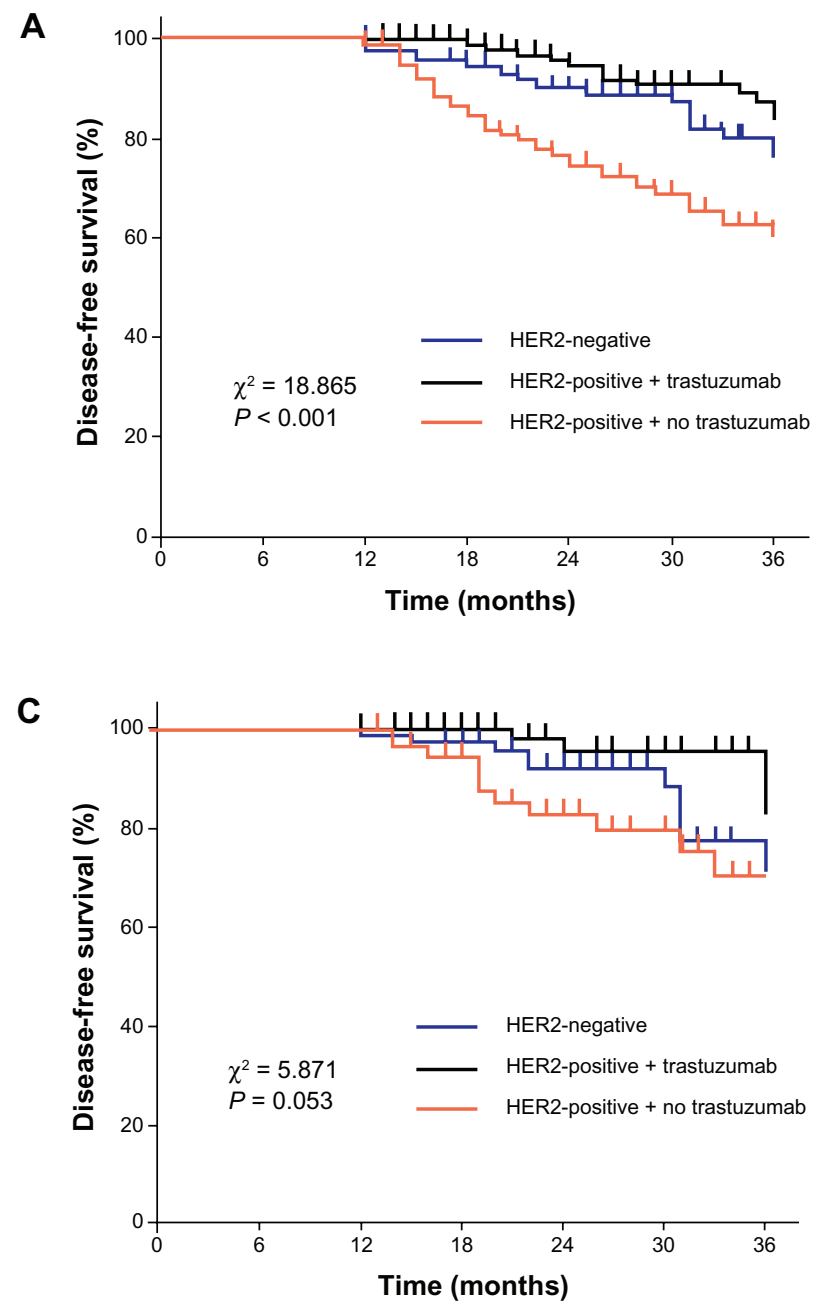

receiving trastuzumab treatment, and the HER2-positive group not receiving treatment were $82.6 \%, 89.6 \%$, and $67.0 \%$, respectively. There was a statistically significant difference in 3-DFS between either of the first two groups and the third group $(P<0.01)$, but the difference between the first two groups was not statistically significant $(P=0.157)$ (Figure 1).

The 3-DFS rates of the three groups during two different periods (2002-2006 and 2007-2010) are illustrated in Figure $1 \mathrm{~B}$ and $\mathrm{C}$; the differences in 3-DFS rate among the three groups were statistically significant for both periods. Analysis showed 3-DFS rates during the period 2002-2006 were similar to those for the whole patients. The HER2positive patients not receiving trastuzumab treatment showed a significantly lower 3-DFS rate than the other two groups $(P<0.05)$. The difference in 3-DFS rate only achieved statistical significance between those receiving and those not receiving trastuzumab treatment within the HER2-positive groups $(P=0.014)$.
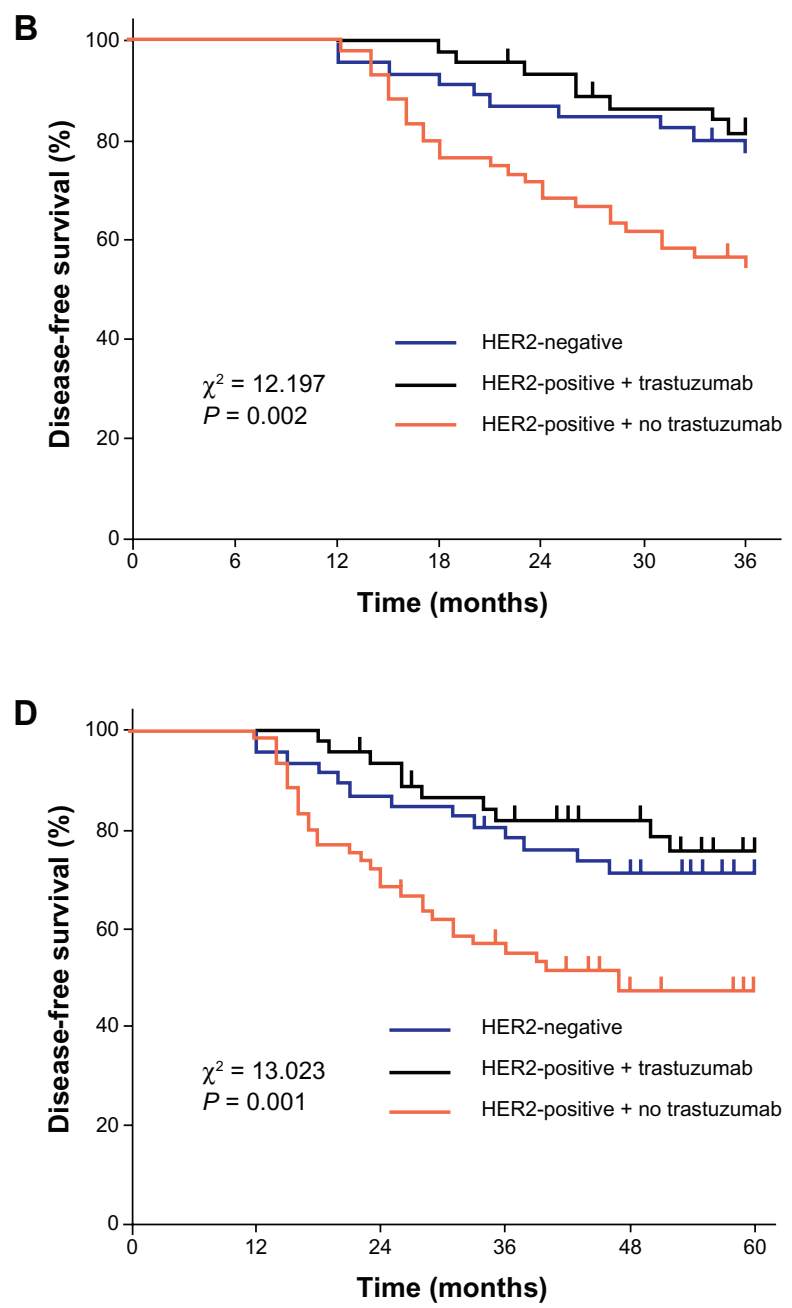

Figure I (A) 3-year DFS during the whole period studied, 2002-2010; (B) 3-year DFS during the period studied, 2002-2006; (C) 3-year DFS during the period studied, 2007-2010; (D) 5-year DFS among three groups during the period studied, 2002-2006. 
The 5-DFS rates during the period 2002-2006 were also analyzed. The 5-DFS rates in the HER2-negative group, the HER2-positive group receiving trastuzumab treatment, and the HER2-positive group not receiving trastuzumab treatment were $71.7 \%, 77.8 \%$, and $48.3 \%$, respectively (Figure 1D). There was a statistically significant difference in 5-DFS between either of the first two groups and the third group $(P<0.05)$, but the difference between the first two groups was not statistically significant $(P=0.503)$.

The 3-DFS rates of the three groups were higher during the period 2007-2010 than during 2002-2006. The 3-DFS rate in the HER2-positive group not receiving trastuzumab treatment increased from $55.0 \%$ in $2002-2006$ to $80.0 \%$ in 2007-2010 (Figure 2A). In the HER2-negative group it increased from $78.3 \%$ to $85.5 \%$ and in the HER2-positive group receiving trastuzumab treatment it increased from $82.2 \%$ to $94.3 \%$ (Figure $2 \mathrm{~B}$ ). However, the differences among the three groups did not achieve statistical significance (HER2-positive group not receiving trastuzumab treatment: $P=0.084$; HER2-negative group: $P=0.902$; HER2-positive group receiving trastuzumab treatment: $P=0.47$ ).

To further explore the differences of 3-DFS among the three groups, a multivariate analysis was performed. This involved the following variables: time period, age, tumor size, tumor grade, lymph node status, hormone receptor status, surgery type, radiotherapy, endocrine therapy, and chemotherapy (Table 2). The results of this multivariate analysis demonstrated that, among others, the significance of the three groups by HER 2 status or by different treatments were predictive for 3-DFS, and the 3-DFS of the HER2-positive group receiving trastuzumab treatment was similar to that of the HER2-negative group.

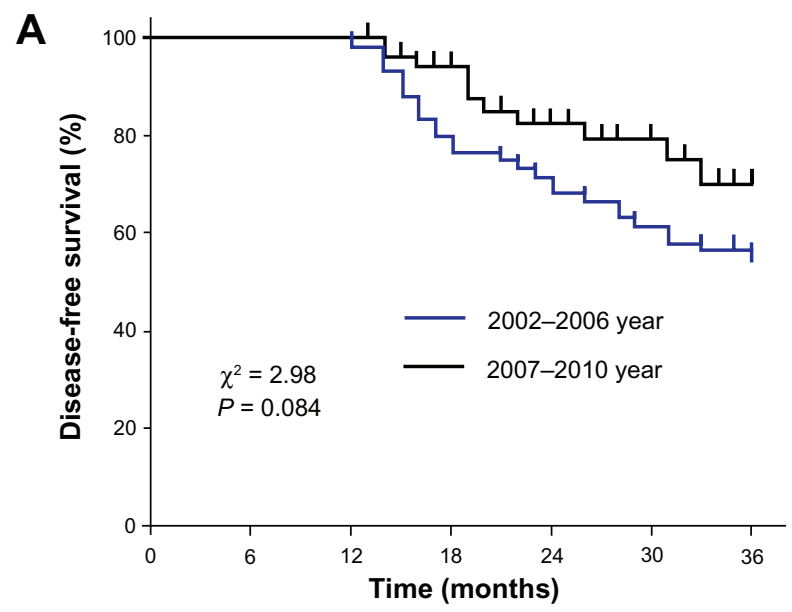

\section{Discussion}

Over the past 10 years, the HER2-based BC classifications have been increasingly used in treatment selection and prognosis prediction, resulting in an improved clinical outcome of BC patients. ${ }^{5-9}$ Nevertheless, treatment efficacy should also be comprehensively evaluated in different regions in order to better understand the potential resistance and variations of HER2-targeted therapy.

The purpose of this study was to compare the clinical outcomes of HER2-positive BC patients receiving or not receiving trastuzumab treatment and HER2-negative BC patients, in a retrospective analysis of operable $\mathrm{BC}$ patients in the People's Republic of China. The results, as outlined, showed that the patients receiving trastuzumab obtained a significantly lower risk of HER2-positive $\mathrm{BC}$ cancer recurrence than the patients not receiving trastuzumab. Also, the 3-DFS and 5-DFS rates in HER2-positive patients receiving trastuzumab treatment were higher than those in HER2-negative patients receiving conventional adjuvant treatment, although the differences were not statistically significant $(P>0.05)$.

In the present study, in multivariate analysis by 3 -DFS this study showed a $68.6 \%$ reduction in risk of recurrence (hazard ratio, $0.314 ; 95 \%$ confidence interval, $0.159-0.619$; $P=0.001)$ in the HER2-positive patients who received trastuzumab treatment when compared with the HER2-positive patients who did not receive trastuzumab treatment. This finding was consistent with the $30 \%-50 \%$ relative risk reduction of recurrence and death previously found in large randomized clinical trials such as BCIRG 006, HERA, N9831, and B31.9-12

Moreover, this study also compared outcome between HER2-positive patients receiving trastuzumab treatment

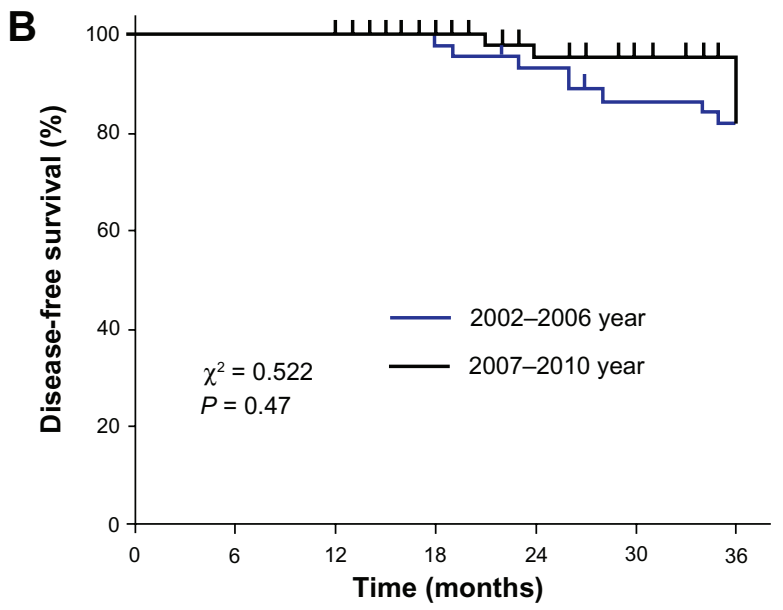

Figure 2 Analysis of 3-year disease-free survival rates between the periods 2002-2006 and 2007-2010 in human epidermal growth factor receptor 2-positive groups (A) not receiving trastuzumab; (B) receiving trastuzumab treatment. 
Table 2 Multivariate analysis of 3-year disease-free survival

\begin{tabular}{|c|c|c|}
\hline Item & Hazard ratio $(95 \% \mathrm{Cl})$ & $P$-value \\
\hline Total study population & - & 0.001 \\
\hline HER2-negative group & Reference* & - \\
\hline $\begin{array}{l}\text { HER2-positive group not } \\
\text { receiving trastuzumab }\end{array}$ & $1.976(1.133-3.445)$ & 0.016 \\
\hline $\begin{array}{l}\text { HER2-positive group } \\
\text { receiving trastuzumab }\end{array}$ & $0.620(0.299-1.286)$ & 0.199 \\
\hline Age & 1.249 (0.744-2.097) & 0.399 \\
\hline Tumor size & I.34I (0.90I-I.996) & 0.148 \\
\hline Tumor grade & $2.03 \mathrm{I}(\mathrm{I} .227-3.36 \mathrm{I})$ & 0.006 \\
\hline Hormone receptors & $0.46 I(0.280-0.760)$ & 0.002 \\
\hline Lymph nodes & $1.415(1.06 \mathrm{I}-\mathrm{I} .888)$ & 0.018 \\
\hline Time periods & $1.089(0.633-1.872)$ & 0.759 \\
\hline Radiotherapy & $0.843(0.470-1.51 \mathrm{I})$ & 0.567 \\
\hline Surgery & $1.033(0.437-2.437)$ & 0.942 \\
\hline Chemotherapy & $0.588(0.238-1.455)$ & 0.251 \\
\hline
\end{tabular}

Note: *HER2-negative used as reference state in multivariate analysis.

Abbreviations: $\mathrm{Cl}$, confidence interval; HER2, human epidermal growth factor receptor 2 .

and HER2-negative patients receiving conventional therapy in the adjuvant setting. The results showed a superior clinical outcome for 3-DFS in the HER2-positive group receiving trastuzumab treatment compared with that in the HER2-negative group, although the differences were not statistically significant $(P>0.05)$ in univariate and multivariate analysis. Although there have not been any large, perspective, randomized studies to compare the prognosis between patients receiving trastuzumab treatment and HER2-negative patients, there have been some small clinical studies that support the results of the present study. For example, the NOAH trial compared the event-free rates of 99 HER2-negative patients with those of HER2-positive patients who either did or did not receive trastuzumab treatment, and the results showed that the event-free rate did not differ between HER2-positive patients receiving trastuzumab treatment and HER2-negative patients. ${ }^{14}$ Another study showed that HER2-positive BC patients receiving trastuzumab treatment had higher survival rates than HER2-negative BC patients. ${ }^{21}$

The authors acknowledge this study has some limitations. First, this study had a small sample size, a retrospective nature, and a short-term follow-up period; a larger, randomized, and prospective study with long-term follow-up should be considered for the future. Second, although the clinicopathologic characteristics of the BC patients in this study were well matched, minimizing the heterogeneities among three groups, there were many other factors such as chemotherapy regimen and the individual economic and social status of patients that could possibly have interfered with the results.

\section{Conclusion}

In summary, the authors evaluated the efficacy of trastuzumab in Chinese HER2-positive BC patients. Not only did this study further confirm the efficacy of trastuzumab therapy in operable HER2-positive BC but also it demonstrated the comparability of 3-DFS and 5-DFS rates between HER2-positive patients receiving trastuzumab treatment and HER2-negative patients receiving conventional adjuvant therapies. From the results of this study, the authors suggest that the prognosis of HER2-positive BC patients receiving trastuzumab is comparable to that of HER2 negative BC patients.

\section{Acknowledgments}

This work was supported by grants from the National Natural Science Foundation of China.(81272896).

\section{Disclosure}

The authors report no conflicts of interest in this work.

\section{References}

1. Jemal A, Bray F, Center MM, Ferlay J, Ward E, Forman D. Global cancer statistics. CA Cancer J Clin. 2011;61(2):69-90.

2. Benson JR, Jatoi I, Keisch M, Esteva FJ, Makris A, Jordan VC. Early breast cancer. Lancet. 2009;373(9673):1463-1479.

3. Goldhirsch A, Ingle JN, Gelber RD, Coates AS, Thürlimann B, Senn HJ. Thresholds for therapies: highlights of the St Gallen International Expert Consensus on the Primary Therapy of Early Breast Cancer 2009. Ann Oncol. 2009;20(8):1319-1329.

4. Peto R, Davies C, Godwin J, et al; Early Breast Cancer Trialists' Collaborative Group. Comparisons between different polychemotherapy regimens for early breast cancer: meta-analyses of long-term outcome among 100,000 women in 123 randomised trials. Lancet. 2012;379(9814):432-444.

5. Wolff AC, Hammond ME, Schwartz JN, et al; American Society of Clinical Oncology; College of American Pathologists. American Society of Clinical Oncology/College of American Pathologists guideline recommendations for human epidermal growth factor receptor 2 testing in breast cancer. J Clin Oncol. 2007;25(1):118-145.

6. Ross JS, Slodkowska EA, Symmans WF, Pusztai L, Ravdin PM, Hortobagyi GN. The HER-2 receptor and breast cancer: ten years of targeted anti-HER-2 therapy and personalized medicine. Oncologist. 2009; 14(4):320-368.

7. Saxena R, Dwivedi A. ErbB family receptor inhibitors as therapeutic agents in breast cancer: current status and future clinical perspective. Med Res Rev. 2012;32(1):166-215.

8. Higgins MJ, Baselga J. Targeted therapies for breast cancer. J Clin Invest. 2011;121(10):3797-3803.

9. Slamon D, Eiermann W, Robert N, et al; Breast Cancer International Research Group. Adjuvant trastuzumab in HER2-positive breast cancer. N Engl J Med. 2011;365(14):1273-1283.

10. Perez EA, Romond EH, Suman VJ, et al. Four-year follow-up of trastuzumab plus adjuvant chemotherapy for operable human epidermal growth factor receptor 2-positive breast cancer: joint analysis of data from NCCTG N9831 and NSABP B-31. J Clin Oncol. 2011;29(25):3366-3373.

11. Gianni L, Dafni U, Gelber RD, et al; Herceptin Adjuvant (HERA) Trial Study Team. Treatment with trastuzumab for 1 year after adjuvant chemotherapy in patients with HER2-positive early breast cancer: a 4-year follow-up of a randomised controlled trial. Lancet Oncol. 2011;12(3):236-244. 
12. Chang HR. Trastuzumab-based neoadjuvant therapy in patients with HER2-positive breast cancer. Cancer. 2010;116(12):2856-2867.

13. Untch M, Rezai M, Loibl S, et al. Neoadjuvant treatment with trastuzumab in HER2-positive breast cancer: results from the GeparQuattro study. J Clin Oncol. 2010;28(12):2024-2031.

14. Gianni L, Eiermann W, Semiglazov V, et al. Neoadjuvant chemotherapy with trastuzumab followed by adjuvant trastuzumab versus neoadjuvant chemotherapy alone, in patients with HER2-positive locally advanced breast cancer (the NOAH trial): a randomised controlled superiority trial with a parallel HER2-negative cohort. Lancet. 2010;375(9712):377-384.

15. Coudert BP, Arnould L, Moreau L, et al. Pre-operative systemic (neo-adjuvant) therapy with trastuzumab and docetaxel for HER2overexpressing stage II or III breast cancer: results of a multicenter phase II trial. Ann Oncol. 2006;17(3):409-414.

16. Buzdar AU, Ibrahim NK, Francis D, et al. Significantly higher pathologic complete remission rate after neoadjuvant therapy with trastuzumab, paclitaxel, and epirubicin chemotherapy: results of a randomized trial in human epidermal growth factor receptor 2-positive operable breast cancer. J Clin Oncol. 2005;23(16):3676-3685.
17. Kwong A, Mang OW, Wong CH, Chau WW, Law SC; Hong Kong Breast Cancer Research Group. Breast cancer in Hong Kong, southern China: the first population-based analysis of epidemiological characteristics, stage-specific, cancer-specific, and disease-free survival in breast cancer patients: 1997-2001. Ann Surg Oncol. 2011;18(11):3072-3078.

18. Wang Y, Yin Q, Yu Q, et al. A retrospective study of breast cancer subtypes: the risk of relapse and the relations with treatments. Breast Cancer Res Treat. 2011;130(2):489-498.

19. Zheng S, Bai JQ, Li J, et al. The pathologic characteristics of breast cancer in China and its shift during 1999-2008: a national-wide multicenter cross-sectional image over 10 years. Int J Cancer. 2012;131(11): 2622-2631.

20. Chen W, Jiang Z, Shao Z, Sun Q, Shen K. An economic evaluation of adjuvant trastuzumab therapy in HER2-positive early breast cancer. Value Health. 2009; 12 Suppl 3:S82-S84.

21. Dawood S, Broglio K, Buzdar AU, Hortobagyi GN, Giordano SH. Prognosis of women with metastatic breast cancer by HER2 status and trastuzumab treatment: an institutional-based review. J Clin Oncol. 2010;28(1):92-98.
OncoTargets and Therapy

\section{Publish your work in this journal}

OncoTargets and Therapy is an international, peer-reviewed, open access journal focusing on the pathological basis of all cancers, potential targets for therapy and treatment protocols employed to improve the management of cancer patients. The journal also focuses on the impact of management programs and new therapeutic agents and protocols on

\section{Dovepress}

patient perspectives such as quality of life, adherence and satisfaction. The manuscript management system is completely online and includes a very quick and fair peer-review system, which is all easy to use. Visit http://www.dovepress.com/testimonials.php to read real quotes from published authors. 